\title{
EDUCANDO PARA LA SALUD - CONOCIMIENTO Y FUENTE DE INFORMACIÓN SOBRE SÍNDROME DE INMUNODEFICIENCIA ADQUIRIDA O SIDA
}

\author{
Maria Helena Bittencourt Westrupp * \\ Cleide Peron Boell Pimente/* \\ Elza Berger Salema Coelho* \\ João Carlos Caetano* \\ Aldanei Norma de Souza **
}

WESTRUPP, M.H.B.; PIMENTEL, C.P.B.; COELHO, E.B.S.; CAETANO, J.C.; SOUSA, A.N. DE. Educando para la salud - conocimiento y fuente de información sobre síndrome de inmunodeficiencia adquirida - SIDA. Rev.latino-am.enfermagem, Ribeirão Preto, v.4, n. 2, p. 61-71, julho 1996

Fueron aplicados 151 cuestionarios a los alumnos de una Escuela Estatal que enseña primaria, secundaria) preparatoria ${ }^{\star \star *}$, con edades entre 10 y 19 años, en Florianópolis en el Estado de Santa Catarina - Brasil. El objetivo era identificar: fuentes de información; mecanismos de transmisión; formas de prevención y el interés en obtener nuevos conocimientos sobre HIVISIDA de los alumnos. Dentro de las respuestas, se descubrió que el $90.06 \%$ escucharon sobre SIDA a través de la televisión, el 29.13\% conocían el tema por el profesor. Se observó también que los padres fueron citados $45.03 \%$ como fuente de referencia para complementar la información y los profesores 9.93\%.

TÉRMINOS CLAVES: SIDA, HIV, estudiantes

\section{INTRODUCCIÓN}

En Brasil hasta diciembre de 1995, fueron notificados un total de 76,396 casos de Síndrome de Inmunodeficiencia Adquirida (SIDA), con una distribución

\footnotetext{
*Departamento de Salud Pública de la Universidad Federal de Santa Catarina - SPB/UFSC

**Estudiante de la Universidad Federal de Santa Catarina y Becaria del Núcleo de Investigación y Extensión de Salud Escolar del Departamento de Salud Pública de la Universidad Federal de Santa Catarina

***Niveles básicos de Educación
} 
de incidencia acumulada especifica (tasa por 100,000 hab.) de 56.3 (BRASIL. Ministério da Saúde, 1995) ${ }^{1}$. Según la Secretaría de Estado de Salud, Santa Catarina registró hasta noviembre de 1995, 1914 casos de SIDA, de los cuales 42.0\% por contaminación por medio del uso de drogas inyectables. Dentro de los usuarios de drogas inyectables, $34.2 \%$ son del sexo masculino y el $7.8 \%$ son del sexo femenino (SANTA CATARINA) ${ }^{8}$. Esto ha contribuido indirectamente para el aumento de casos de SIDA entre los heterosexuales, particularmente en mujeres con edad fértil.

Así, como en otros países, y en los demás estados brasileños, también en Santa Catarina, el SIDA y la infección por Virus de Inmunodeficiencia Humana (VIH), ha llamado la atención por los problemas sociales, culturales, económicos, políticos, éticos y jurídicos.

Florianópolis, localizada en la mayor isla del litoral del Estado de Santa Catarina, se constituye en un centro turístico de la región sur del continente sudamericano, por su situación geográfica, las bellezas naturales y la hospitalidad de la población.

Tiene aproximadamente 260.170 habitantes y es el municipio con mayor registro de casos de SIDA (509), hasta diciembre de 1995, contribuyendo para que Santa Catarina "debido a su localización, el crecimiento de la demanda de turistas y de la población, tenga su historia en relación al SIDA, marcada como la epidemia del final de este siglo y que en comparación con los estados del sur el aumento de casos de la enfermedad presenta números significativos". (WESTRUPP et al., 1994) ${ }^{9}$

Por esta razón, se buscó junto a los estudiantes de primaria, inscritos en una Escuela Estatal su conocimiento y la información sobre VIH/SIDA, y con los datos presentados, desarrollar un trabajo basado en los principios de educación participativa, por la Escuela y el Departamento de Salud Pública (SPB) de la Universidad Federal de Santa Catarina (UFSC). Esta investigación acerca de SIDA, hace parte de un Proyecto de Educación y Salud, con estudiantes que necesitan la identificación, la reflexión y acciones educativas que contribuyan para la resolución de problemas encontrados junto a los estudiantes.

\section{ÁREA DE ESTUDIO}

La Escuela Estatal Getúlio Vargas, ubicada en el Barrio de Saco de los Limones, en Florianópolis-SC, tiene capacidad para 2,500 alumnos y ofrece preescolar y primaria completando el primer ciclo de educación. Ofrece, además, cursos para formación de técnicos de administración, diseñador de instalaciones eléctricas, curso de magisterio y educación general como la preparación para el examen de ingreso a la Universidad. En convenio con el ayuntamiento de Florianópolis, la escuela implantó un curso de alfabetización de jóvenes y adultos. 


\section{METODOLOGIA}

La investigación fue realizada con alumnos de 5ta. hasta 8va. fase de primaria de la Escuela Estatal Getúlio Vargas.

La selección de la Escuela, de los alumnos y del estudio, fue basada en lo siguiente: 1) La Escuela es parte de la área de extensión del Núcleo de Investigación y Extensión en Salud Escolar (NUPESE), del Departamento de Salud Pública de la Universidad Federal de Santa Catarina; 2) Su población es de adolescentes que inician su actividad sexual o están en ejercicio de la misma; 3) Es necesario trabajar cada vez más éste tema con jóvenes en esta fase de la vida, en la medida de las posibilidades del Núcleo, junto con los padres, y la Escuela que también son responsables por la orientación y la formación de los mismos; 4) El estudio es parte del proyecto de Educación y Salud del Núcleo de Investigación y Extensión en Salud Escolar (NUPESE) del Departamento de Salud Pública de la Universidad Federal de Santa Catarina.

El estudio se realizó en tres etapas:

\section{$1^{\text {a }}$ Etapa:}

Fase de integración entre el personal que labora en la Escuela y los investigadores: fue realizada por medio de reuniones con profesores, orientadores y director de la escuda. Con los estudiantes fue discutido en clase el objetivo del trabajo y se les aplicó un formulario con las siguientes preguntas:

a) ¿Qué entiende por Salud?

b) ¿Cuáles son los problemas de salud de la escuela?

c) ¿Qué desearía saber sobre salud?

\section{$2^{\text {a }}$ Etapa:}

Dentro de la pregunta qué desearían saber sobre salud; el tema del SIDA fue uno de los más solicitados. Para trabajar con el tema, se aplicó un nuevo cuestionario con preguntas acerca de las características personales, con la finalidad de describir la población (sexo, edad) y preguntas para detectar qué información tenían sobre:

a) Fuente de información.

b) Agente, medio de transmisión y formas de prevención del SIDA.

c) Interés en obtener otra información.

d) A quién acudiría para obtener otra información.

Al analizar esta etapa surge el presente trabajo. 


\section{$3^{a}$ Etapa:}

Se encuentra en desarrollo y hasta hoy se han presentado videos educativos sobre SIDA y están siendo desarrolladas tallares de trabajo sobre el asunto.

El formulario fue aplicado en 1994, a 151 alumnos presentes, en el momento de la selección de los datos, cerca de $20.21 \%$, de los estudiantes matriculados en 1994, de 5ta. hasta 8va. fase de primaria.

Los resultados son variados en concordancia a la variación del número de las respuestas obtenidas.

\section{CARACTERÍSTICAS DE LA POBLACIÓN}

Se seleccionó una muestra de 151 alumnos, inscritos en 1994 de los cuales $20.21 \%$, eran de $5 \mathrm{ta}$. hasta $8 \mathrm{va}$. fase de primaria; con edades entre 10 y 19 años siendo la mayoría, $73.52 \%$ de 11 a 13 años y $54.96 \%$ eran de sexo masculino. (Tabla 1 )

TABLA 1 - DISTRIBUCIÓN DE LOS ESTUDIANTES, SEGÚN LA EDAD Y EL SEXO - FLORIANÓPOLIS - SC - 1994

\begin{tabular}{|c|c|c|c|c|c|c|}
\hline \multirow{2}{*}{$\begin{array}{l}\text { SEXO } \\
\text { EDAD }\end{array}$} & \multicolumn{2}{|c|}{ MASCULINO } & \multicolumn{2}{|c|}{ FEMININO } & \multicolumn{2}{|c|}{ TOTAL } \\
\hline & N. ${ }^{\circ}$ & $\%$ & N. ${ }^{\circ}$ & $\%$ & N. ${ }^{\circ}$ & $\%$ \\
\hline 10 & 04 & 2.65 & 02 & 1.32 & 06 & 3.97 \\
\hline 11 & 15 & 9.93 & 20 & 13.25 & 35 & 23.18 \\
\hline 12 & 17 & 11.26 & 14 & 9.28 & 31 & 20.54 \\
\hline 13 & 24 & 15.90 & 21 & 13.90 & 45 & 29.80 \\
\hline 14 & 09 & 5.96 & 06 & 2.98 & 15 & 9.94 \\
\hline 15 & 10 & 6.62 & 03 & 1.99 & 13 & 8.61 \\
\hline 16 & 01 & 0.66 & 02 & 1.32 & 03 & 1.98 \\
\hline 17 & 01 & 0.66 & - & - & 01 & 0.66 \\
\hline 18 & - & & - & - & - & - \\
\hline 19 & 02 & 1.32 & - & - & 02 & 1.32 \\
\hline TOTAL & 83 & 54.96 & 68 & 45.04 & 151 & 100,00 \\
\hline
\end{tabular}




\section{RESULTADOS Y DISCUSIÓN}

Al analizar los medios de información, a los cuales la población estudiada tiene acceso para obtener algún conocimiento sobre SIDA, se verificó que los 151 alumnos mencionaron más de un medio de información; los más citados por los estudiantes fueran: $90.06 \%$ la televisión, $43.70 \%$ los familiares y $29.13 \%$ los profesores (Tabla 2).

TABLA 2 - MEDIOS DE INFORMACIONES SOBRE SIDA CITAOS POR LA POBLACIÓN EN ESTUDIO - FLORIANÓPOLIS - SC - 1994

\begin{tabular}{c|c|c}
\hline MEDIO DE INFORMACIÓN & $\mathbf{N}^{\mathbf{0}^{\circ}}$ & $\%$ \\
\hline TELEVISIÓN & 136 & 90.06 \\
RADIO & 48 & 31.78 \\
PROFESORES & 44 & 29.13 \\
FAMILIARES & 66 & 43.70 \\
AMIGO & 47 & 31.12 \\
REVISTA & 76 & 50.33 \\
\hline
\end{tabular}

$\mathrm{N}=$ Número de veces que cada medio de información fue citado por los 151 estudiantes

El porcentaje de $43.70 \%$ y de $29.13 \%$ alcanzados por los familiares y profesores, respectivamente, deben tener más consideración, por el papel fundamental de orientación que desempeñan también en la enseñanza para la prevención del SIDA, por los datos presentados es menor que el índice relacionado a la televisión; en nuestro entendimiento, este medio de comunicación, como fuente de información deja mucho que desear, teniendo en cuenta que la información transmitida por los medios de comunicación, frecuentemente tienen preconceptos. Además los programas transmitidos (novelas y programas humorísticos) a los cuales, los jóvenes tienen acceso, en su mayoría son abordados de forma sarcástica y guiados por machismo latino-americano.

"Una de las medidas más importantes de la profilaxis de las enfermedades transmisibles es la educación para la salud" (Amato Neto apud KAMIYAMA, 1978) ${ }^{3}$. En relación al SIDA como se sabe, por lo menos hasta el momento no hay duda de que una forma más eficaz para la prevención es la actividad educativa.

En lo que se refiere al carácter da transmisibilidad de SIDA, 93.37\% afirmaron es una enfermedad sexualmente transmisible, $2.00 \%$ no la reconocen como enfermedad transmisible y $4.63 \%$ no respondieron. (Tabla 3 ) 
TABLA 3 - DISTRIBUCIÓN DE LOS 151 ESTUDIANTES SEGÚN EL CONOCIMIENTO SOBRE LA TRANSMISIÓN DE SIDA - FLORIANÓPOLIS - SC 1994

\begin{tabular}{l|c|c}
\hline \multicolumn{1}{c|}{$\begin{array}{c}\text { CONOCIMIENTO DE LA } \\
\text { TRANSMISIÓN }\end{array}$} & N. $^{\circ}$ & $\%$ \\
\hline SI & 141 & 93.37 \\
NO & 03 & 2.00 \\
NO RESPONDIDO & 07 & 4.63 \\
\hline TOTAL & $\mathbf{1 5 1}$ & $\mathbf{1 0 0 . 0 0}$ \\
\hline
\end{tabular}

Cuando preguntamos sobre cuál es el agente causador de SIDA, 60.26\% respondieron que es por el $\mathrm{VIH} ; 24.50 \%$ relacionaron que es por el "sexo, jeringa que no se cambian de aguja, droga inyectada en la vena"; por "sexo sin condón" $7.28 \%$ de las respuestas; "no saben" $3.31 \%$ y $4.65 \%$ respondieron lo siguiente: "son las mujeres, creo que son los monos y que los científicos inventaron el SIDA" (Tabla 4).

TABLA 4 - AGENTE QUE CAUSA SIDA SEGÚN LA POBLACIÓN ESTUDIADA FLORIANÓPOLIS - SC - 1994

\begin{tabular}{l|c|c}
\hline \multicolumn{1}{c|}{ AGENTE } & N. $^{{ }^{\circ}}$ & $\%$ \\
\hline HIV & 91 & 60.26 \\
SEXO, JERINGA Y DROGA & 37 & 24.50 \\
RELAC. SEXUALES S/ CONDÓN & 11 & 7.28 \\
NO SABE & 05 & 3.31 \\
OTRAS RESPUESTAS & 07 & 4.65 \\
\hline
\end{tabular}

$\mathrm{N}=$ Número de veces que cada agente de referencia fue citado por los 151 estudiantes

Estas respuestas muestran la diversidad de información a la que los adolescentes están sujetos, no teniendo, en algunos casos, conocimiento correcto sobre la pregunta. "Los jóvenes adquieren del medio la percepción de si mismos. Ellos se orientan por aquello que los otros dicen, por la manera que reaccionan sus impulsos, la forma de tratarlos, en el modo de ayudarlos para que se integren a las nuevas necesidades" (Carballo apud LINS, 1988) ${ }^{4}$. 
En el momento en que fueron realizadas las encuestas se reconocía el VIH como causador del SIDA, $79.47 \%$ respondieron que si, encuanto que $15.23 \%$ no lo reconocieron y $5.29 \%$ no respondieron. Estos datos demuestran la necesidad de abordar la prevención con información que puede producir cambio, no solo de conocimientos, sino también de una modificación efectiva de comportamiento de los adolescentes.

Sobre los medios de transmisión del SIDA, conocidos por los alumnos, 83.44\% citaron la transmisión sexual, y cuando se les preguntó sobre el conocimiento de alguna forma de prevenirse, $90.73 \%$ dijeron que conocían, $5.30 \%$ respondieron que no tenían conocimiento y 3.97 no respondieron.

De las formas de prevención conocidas, $85,43 \%$ de los alumnos mencionaron el uso de condón.

"Según algunos educadores que trabajan con SIDA, es mucho más fácil enseñar prácticas sexuales seguras, incluyendo el uso de preservativos a los jóvenes que no han desarrollado práctica de riesgo. Hablar para niños al mismo tiempo también es un medio de discutir los papeles de género antes que estos influencien en decisiones relacionadas a la vida sexual" (O'LEARY \& CHENEY, 1993) ${ }^{5}$.

En el conjunto de las respuestas dadas, (Tabla 5) la población en estudio mencionó 250 formas de prevención, 02 no sabe como prevenir y 10 no respondieron.

CORDEIRO et al. (1994) obtienen respuestas semejantes, en investigaciones realizadas con alumnos del curso universitario -y CORDEIRO et al. (1994) cita DAWSON; DICLEMENTE; GOODMAN, que también obtuvieron respuestas iguales en investigaciones realizadas con adolescentes.

TABLA 5 - FORMAS DE PREVENIR EL SIDA SEGÚN LA POBLACIÓN ESTUDIADA - FLORIANÓPOLIS - SC - 1994

\begin{tabular}{l|c|c}
\hline \multicolumn{1}{c|}{ FORMAS DE PREVENIR } & $\mathbf{N .}^{\mathbf{0}^{*}}$ & \% \\
\hline USO DE CONDÓN & 129 & 49.24 \\
NO USANDO DROGAS O LA MISMA JERINGA & 69 & 26.33 \\
NO HACER TRANSFUSIÓN DE SANGRE & 07 & 2.67 \\
NO TENER RELACIONES SEXUALES CON EXTRAÑOS & 06 & 2.30 \\
EVITAR RELACIONES SEXUALES & 07 & 2.67 \\
NO TENER RELACIONES SEXUALES CON PORTADORES DE & 06 & 2.30 \\
EST/SIDA & & \\
OTRAS RESPUESTAS & 26 & 9.92 \\
NO SABE & 02 & 0.76 \\
NO RESPONDIDO & 10 & 3.81 \\
\hline
\end{tabular}

$\mathrm{N}=$ Número de veces en que las formas de prevenirse fueron citadas por los 151 estudiantes 
EL uso de condón fue citado en $49.24 \%$ y no usando drogas ni la misma jeringa en $26.33 \%$ de las respuestas.

Como otras respuestas, $9.92 \%$ mencionaron como formas de prevención tener pocas relaciones sexuales, pedir certificado médico, no prestar objetos íntimos y no hacer sexo antes del casamiento.

Esta percepción también fue constatada en investigación realizada (PIMENTEL et al., 1992) ${ }^{6}$ con adolescentes de una escuela pública municipal de Florianópolis, sobre enfermedades sexualmente transmisibles, al describir que en relación a los medios de prevención, consiguieron relacionarla al uso de condón, y algunas formas no usuales, tales como píldora anticonceptiva, no tener relaciones ilícitas, ser responsable, y uno respondió no tener relaciones sexuales.

Un estudio lecho por CORDEIRO et al. (1994), muestra que entre los estudiantes universitarios "Los revistas, Los periódicos y La televisión constituyen las principales fuentes de información sobre SIDA. "Comparando las respuestas dadas en las dos investigaciones realizadas, se verifica que la referencia entre los adolescentes, para la búsqueda de información es la familia (padre, madre, familiares) contrariamente las respuestas dadas por los universitarios. Esto nos hace pensar que se debe a que los adolescentes, en su mayoría, viven junto a sus familiares, en cuanto los universitarios, al estar, normalmente, lejos de sus familias buscan otras fuentes de información. Mientras tanto, la información es apenas uno de los componentes del proceso educativo en la prevención del SIDA. A pesar de las campañas informativas que están siendo realizadas, han aumentado progresivamente el número de enfermos infectados por el VIH. Así, se considera urgente la realización de investigaciones sobre factores psicosocio-culturales, posibles determinantes de conducta sexual de riesgo, con la finalidad de mejorar la dirección de los trabajos educativos para la prevención del SIDA" (CORDEIRO et al. 1994).

Se les pregunto si desearían saber más sobre el asunto, 75.49\% respondieron que si, porque sienten necesidad de obtener más información; $19.21 \%$ no manifiestan interés y $5.30 \%$ no respondieron.

Mientras tanto analizamos las 217 respuestas emitidas, donde se verificó (Tabla 6), que los padres fueron citados en $45.03 \%$ de las respuestas; los amigos $10.60 \%$; los profesores $6.91 \%$; los medios de comunicación (periódicos, revistas, libros, TV) 11.07\%. 
TABLA 6 - FUENTE DE REFERENCIA, PARA COMPLEMENTAR LA INFORMACIÓN SOBRE SIDA, CITADA POR EL ESTUDIANTES INVESTIGADOS FLORIANÓPOLIS - SC - 1994

\begin{tabular}{l|c|c}
\hline \multicolumn{1}{c|}{ FUENTE DE REFERENCIA } & $\mathbf{N}^{\circ}$ & $\%$ \\
\hline PADRES (MADRE, PADRE) & 68 & 45.03 \\
FAMILIARES & 29 & 9.20 \\
AMIGOS & 25 & 16.55 \\
PROFESORES & 15 & 9.93 \\
MEDIOS DE COMUNICACIÓN (RADIO, TV, PERIÓDICOS, & 24 & 15.89 \\
LIBROS) & 17 & 11.25 \\
SERVICIOS DE SALUD (HOSPITALES, CENTROS DE SALUD, & & \\
DOCTOR) & 14 & 9.27 \\
NO RESPONDIDO & 09 & 5.96 \\
NO SABE & 15 & 9.93 \\
OTROS
\end{tabular}

$\mathrm{N}=$ Número de veces en que cada fuente de referencia fui citado por 151 estudiantes

Es importante resaltar que las madres en $11,76 \%$ y los padres en $11,31 \%$, fueron los más citados entre los parientes que serían procurados para buscar más información.

"La participación de adolescentes como multiplicador junto a sus compañeros es un medio efectivo de promover conocimiento, detectar situaciones, discutir el proceso de adolescencia, favoreciendo, de esta forma la valorización de el mismo y de su grupo" (BRASIL. Ministério da Saúde, 1989)².

\section{CONCLUYENDO}

Al analizar los resultados de investigación, observamos que el conocimiento de los alumnos envueltos es cuantitativamente considerable, al ser cuestionados sobre SIDA, 100\% demostraron conocimiento previo. Los medios de comunicación citados desempeñan un papel importante. Como fuente de información, este dato demuestra que los medios de comunicación tienen un papel significativo en detrimento del papel de la familia y de la escuela. 
Los alumnos demuestran necesidad de información adicional sobre el tema. De esa constatación, es necesario establecer una práctica educativa, de forma sistemática, relacionada al SIDA, en las Instituciones de Enseñanza y Salud.

Los servicios de salud y las instituciones escolares necesitan estar debidamente preparados para su papel educativo, desapareciendo los tabús y los estigmas relacionados no solo al SIDA, sino también a la sexualidad humana y las enfermedades sexualmente transmisibles.

\section{HEALTH EDUCATION - KNOWLEDGE AND INFORMATION SOURCES ON AIDS}

Questionnaires were answered by 151 students, 10 to 19 years old, from an elementary, Jr. and Senior - high State School in Florianópolis, State of Santa Catarina -Brazil. The objectives were: to identify information sources; transmission mechanisms; prevention forms and the interest shown by students in acquiring new knowledge on HIVIAIDS. Based on the responses, author found out that 90,06\% of the students had heard about AIDS on TV, 29, 13\% had been informed about the theme through a teacher. It was also observed that $45,03 \%$ would seek for orientation from their parents, and 9,93\%from their teachers.

UNITERMS: AIDS, HIV, students

\section{REFERENCIAS BIBLIOGRAFICAS}

01. BRASIL. Ministério da Saúde - Programa Nacional de Doenças Sexualmente Transmissíveis. AIDS. Brasília. Boletim Epidemiológico. Ano 8, n. 2 - Semana Epidemiológica - 02 dezembro de 1995.

02. BRASIL. Ministério da Saúde - Secretaria Nacional de Programas Especiais de Saúde. Divisão Nacional de Saúde Materno Infantil. Bases programáticas. Brasília, 1989. p. 24.

03. CORDEIRO, R.G.F.; NITRINI, S.M.O.O.; TEMPORINI, E.R. Conhecimentos, opiniões e conduta em relação à AIDS entre estudantes de Farmácia Bioquímica do Estado do Paraná, Brasil. Rev. Bras. Saúde Esc., v. 3, n. 1-4, p. 49-56, 1994. 
04. KAMLYAMA, Y. et al. Ensino de Enfermagem em doenças sexualmente transmissíveis: experiência. Rev. Bras. Enfermagem, v. 31, p. 55-9, 1978.

05. LINS, L.C.S. et al. Como anda a educação sexual dos jovens. Brasília. Rev. Bras. Enfermagem. v. 41, n. 2, p. 123-31, abr./jun. 1988.

06. O'LEARY, 5.; CHENEY, B. (org.). A tripla ameaça: mulheres \& AIDS. (Triple jeopardy). Trad. Ana Dourado. Rio de Janeiro: ABIA, 1993. p. 126.

07. PIMENTEL, C.P.B. et al. Estudo de conhecimento de doenças sexualmente transmissíveis num grupo de adolescentes, de Escola Municipal de Florianópolis. Rev. Ciênc. Saúde. Florianópolis, v. 11, n. 2, p. 226-32, 1992.

08. SANTA CATARINA. (Estado). SECRETARIA DE ESTADO DA SAÚDE. Boletim Epidemiológico da AIDS, 1984 a 09.11.95.

09. WESTRUPP. M.H.B. et al. Santa Catarina diante dos números da AIDS. 1994 (Trabalho para cumprir requisitos da Disciplina do Curso de Doutorado em Filosofia da Enfermagem da Universidade Federal de Santa Catarina) 